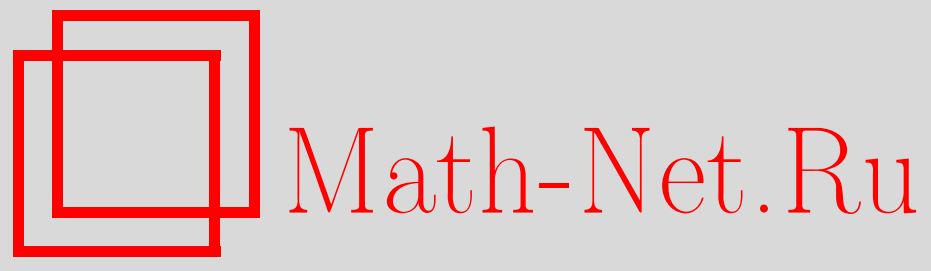

С. Дворянинов, В. Соловьев, Архимед и астрофизика, Квант, 2020, номер 9, 2-9

DOI: https://doi.org/10.4213/kvant20200901

Использование Общероссийского математического портала Math-Net.Ru подразумевает, что вы прочитали и согласны с пользовательским соглашением http://www.mathnet.ru/rus/agreement

Параметры загрузки:

IP: 3.89 .185 .249

26 апреля 2023 г., 16:04:08

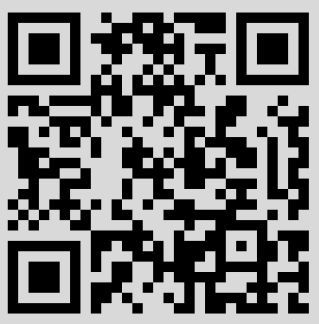




\section{Архимед и астрофизика}

\section{С.ДВОРЯНИНОВ, В.СОЛОВЬЕВ}

\section{Ставим Солнцу градусник}

Популярный миф об Архимеде, бегущем по главной улице Афин с криком «Эврика!», знают все. Как и приписываемое ему же изречение: «Дайте мне точку опоры, и я переверну земной шар!» Будем считать, что на самом деле так все и было, именно так он и говорил. А ведь Архимед был прав: его открытия имеют космический размах и объясняют, в первом приближении, поведение типичных звезд, называемых звездами Главной последовательности. Так что совсем не зря именем Архимеда названа лучшая из опер, написанных студентами физического факультета МГУ.

Наблюдения астрономов, которым как минимум две тысячи лет и которым можно доверять, доказывают, что большинство звезд кажутся вечными и неизменными. А это значит, что к

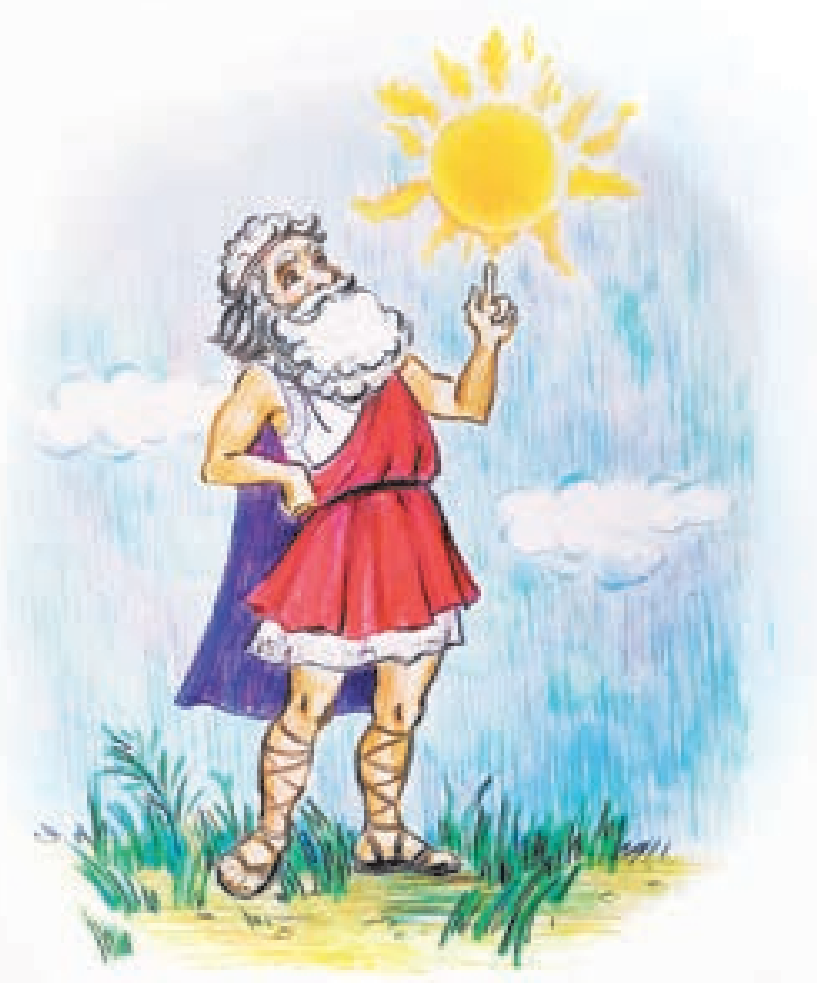
ним применимы законы статики, или равновесия, в которых Архимед давно разобрался. Школьное изложение закона Архимеда сводит его к условию плавания тела в жидкости, причем подсознательно мы подразумеваем под этой жидкостью воду и, конечно, считаем ее несжимаемой. Так сложилось исторически с античных времен. Потом появилось воздухоплавание, и закон Архимеда распространился на газы. Состоят ли звезды из воды? Конечно, нет, а если бы и состояли, то вода точно не оставалась бы в жидком состоянии, а превратилась бы в пар. Применим

ли закон Архимеда к пару? Да, конечно. Если вы хотя бы год изучали в школе физику, то знаете, что этот закон можно перевести на чуть более современный язык Паскаля и Ньютона. И для газа, и для жидкости силы, действующие внутри вещества, описываются давлением, которое не зависит от направления. Это закон Паскаля. Но если это вещество находится в состоянии покоя по отношению к телу огромной массы, например к планете или звезде, то силы давления в нем должно уравновешивать и силу тяготения. А если эта планета или звезда еще и вращается вокруг своей оси, то надо учитывать и 
центробежную силу инерции, но здесь мы о ней ради простоты забудем.

Простейший вариант задачи о давлении внутри несжимаемой жидкости в однородном поле тяжести приводит к известной формуле, применяемой чаще всего к определению давления в воде на глубине $h$ :

$$
p=p_{\mathrm{a}}+\rho g h .
$$

Здесь $p_{\text {a }}$ - это давление атмосферного воздуха у поверхности воды, $g$ - ускорение свободного падения, $h$ - глубина, на которой нас интересует давление, $\rho-$ плотность воды. Формула получается как условие обращения в ноль векторной суммы всех сил, действующих на небольшой объем жидкости, взятый в форме цилиндра или прямой призмы. Это сила тяжести и силы давления на поверхности, ограничивающие выделенный объем.

Пусть теперь вместо воды будет звездное вещество, для него уместно название «плазма». Это газ, в котором электроны оторваны от ядер, объект намного более сложный, чем идеальный газ, с которым вас знакомят на уроках физики. Но для статичной, т.е. неподвижной в среднем, плазмы сложностями можно пренебречь. По сравнению с приведенной школьной формулой давления кое-что все-таки изменится: не только давление $p$, но и все величины, т.е. и ускорение свободного падения $g$ и плотность $\rho$, теперь будут зависеть от глубины, а лучше сказать - от расстояния до центра звезды. А вот усложнения, которые внес в теорию гравитации Эйнштейн, для типичных звезд из Главной последовательности можно не учитывать, так как их гравитационное поле слабое (в сравнении c нейтронными звездами или черными дырами).

Итак, выделим внутри статической (т.е. равновесной) звезды объем вещества в форме цилиндра с площадью оснований $\Delta S$ и высотой $h=\Delta r$. Пусть ось цилиндра лежит на радиусе звезды. Тогда нижнее основание цилиндра отстоит от центра звезды на расстояние $r$, а верхнее - на $r+h=r+\Delta r$. Сила давления на нижнее основание направлена «вверх», т.е. вдоль радиуса от центра звезды, и равна $p(r) \Delta S$, а на верхнее - направлена «вниз» к центру звезды и равна $p(r+\Delta r) \Delta S$. Разность этих величин, равная $-\Delta p \Delta S$, и дает «выталкивающую силу» Архимеда, действующую на цилиндр. Заметьте: функция $p=p(r)-$ функция убывающая. Поскольку вещество цилиндра находится в равновесии (как и все вещество звезды в нашей модели), то цилиндр «плавает» и действующая на него сила тяжести равна выталкивающей силе. Сила же тяжести определяется массой $M(r)$ и радиусом $r$ той части звезды, которая находится ниже нижнего основания цилиндра, а также массой $\Delta m$ вещества, содержащегося внутри цилиндpa:

$$
F=\frac{G M(r) \Delta m}{r^{2}}, \Delta m=\rho(r) \Delta S \Delta r .
$$

Здесь нужно считать величину $\Delta r$ малой, иначе формулы будут сложнее, а выгоды от этого никакой не будет. (Разумеется, это не что иное как стандартный прием исчисления бесконечно малых, изобретенного Ньютоном. Надо сказать, что некоторые результаты Архимеда, например вычисления объемов, показывают, что он и тут был в курсе дела.)

Итак, условием равновесия вещества в цилиндре является равенство

$$
(p(r)-p(r+\Delta r)) \Delta S=\frac{G M(r) \rho(r)}{r^{2}} \Delta S \Delta r,
$$

или

$$
\frac{p(r+\Delta r)-p(r)}{\Delta r}=-\frac{G M(r) \rho(r)}{r^{2}} .
$$

Заметим, что левая часть этого уравнения при малых значениях $\Delta r$ называется производной давления по радиальной координате $r$. Тогда получаем уравнение

$$
p^{\prime}(r)=-\frac{G M(r) \rho(r)}{r^{2}} .
$$

Из астрономических наблюдений обычно можно определить три величины: массу звезды $M$, ее радиус $R$ и ее светимость $L$. Через них вычисляют температуру $T$ на поверхности звезды. Вот и мы уравнение, полученное применением закона Архимеда к физике равновесных звезд, использу- 
ем теперь для оценки давления и температуры в центре звезды. Рассмотрим это уравнение при сильно упрощающих расчеты предположениях, в частности будем считать плотность звезды одинаковой во всем ее объеме. В таком случае

$$
\rho(r)=\frac{M}{V}, V(R)=\frac{4}{3} \pi R^{3},
$$

масса шара радиусом $r$ равна

$$
M(r)=\rho(r) \cdot \frac{4}{3} \pi r^{3}=\frac{M r^{3}}{R^{3}},
$$

а наше уравнение принимает вид

$$
p^{\prime}(r)=-\frac{4 \pi G M^{2}}{3 V^{2}} r .
$$

Интегрируя производную $p^{\prime}(r)$, находим функцию

$$
p(r)=-\frac{2 \pi G M^{2}}{3 V^{2}} r^{2}+c .
$$

Произвольную постоянную $c$ найдем, считая давление на поверхности звезды, т.е при $r=R$, равным нулю. Тогда

$$
c=\frac{2}{3} \frac{\pi G M^{2}}{V^{2}} R^{2} \text { и } p(r)=\frac{2}{3} \frac{\pi G M^{2}}{V^{2}}\left(R^{2}-r^{2}\right) .
$$

При $r=0$ находим давление в центре звезды:

$$
p(0)=\frac{2}{3} \frac{\pi G M^{2}}{V^{2}} R^{2} .
$$

Подставив сюда выражение объема шара через его радиус, получим

$$
p(0)=\frac{3}{8 \pi} \frac{G M^{2}}{R^{4}} .
$$

Например, для Солнца $M \approx 2 \cdot 10^{30}$ кг, $R \approx 7 \cdot 10^{8}$ м и получается $p(0) \approx 10^{14}$ Па, т.е. миллиард атмосфер(!).

Для оценки температуры в центре Солнца используем уравнение состояния идеального газа, т.е. уравнение МенделееваКлапейрона. Газ будем считать атомарным водородом, у которого молярная масса равна $\mathrm{M}=10^{-3}$ кг/моль. Тогда

$$
T(0)=\frac{\mathrm{M} p(0)}{R \rho} \text {. }
$$

Средняя плотность Солнца $\rho=M / V \approx$ $\approx 1,4 \cdot 10^{3} \mathrm{\kappa г} / \mathrm{m}^{3}, \quad R=8,31$ Дж/(К $\cdot$ моль $)$, получается $T(0) \approx 1,2 \cdot 10^{7} \mathrm{~K}$.

Заметим, что согласно современным данным температура в центре Солнца достигает 15 миллионов градусов, а давление составляет $3,4 \cdot 10^{16}$ паскалей. Опираясь лишь на школьную физику (Архимед, Паскаль, Ньютон, Менделеев и Клапейрон), в оценке температуры в центре Солнца мы получили правильный порядок величины, ошибка составляет $\approx 20 \%$. А вот давление получилось на два порядка меньше, чем оно есть на самом деле. Это значит, что плотность в центре Солнца примерно в сто раз больше средней.

Предположения о зависимости плотности звездного вещества от глубины могут быть самыми разными. Далее мы обсудим один экстремальный вариант.

\section{Чудеса гравитации}

Если энергия характеризует способность совершать работу, то отрицательная энергия - это что? Неспособность совершать работу? А вот и не так! Ваше богатство измеряется отрицательной величиной Вы должник! Можете ли Вы тратить деньги? А почему бы нет? Некоторые всю жизнь так и делают. Вспомним, например, Павла Иваныча Чичикова...

В школьных задачах механическая энергия - и кинетическая, и потенциальная обычно положительна. Перепад высот в плотине ГЭС (ничтожно малый в космических масштабах) обеспечивает превращение положительной потенциальной энергии воды в электрическую. Заметим, что любая потенциальная энергия замкнутой системы тел складывается из суммы потенциальных энергий взаимодействий всех пар этих тел. Эту энергию нельзя разделить на индивидуальные порции. Так, например, упомянутая выше потенциальная энергия воды наверху плотины есть на самом деле энергия взаимодействия двух тел: воды и планеты Земля.

В привычной ситуации на Земле при малых высотах и малых перемещениях мы, упрощая, говорим, что потенциальная энергия тела массой $m$ выражается линей- 
ной функцией: $E=m g h$. Мы не упоминаем, что и Земля в равной степени может претендовать на эту энергию. Соломоновым решением было бы деление потенциальной энергии между ними пополам. Но это неверно! Мы считаем, что вся общая потенциальная энергия превращается в кинетическую энергию только одного из двух тел. Почему же мы пренебрегаем приращением кинетической энергии Земли? Потому, что несравнимы массы двух тел, а приобретенные ими импульсы должны быть одинаковыми. Тогда кинетичес-

кая энергия, приобретенная Землей, будет $E_{\text {Земли }}=\frac{p^{2}}{2 M}$, что катастрофически мало по сравнению с кинетической энергией $E_{\text {воды }}=\frac{p^{2}}{2 m}$, которую получает вода.

Обычно $h$ - это высота, отсчитываемая от определенного уровня, например от поверхности Земли в данном месте, а $g \approx G \frac{M}{R^{2}}$, где $M-$ масса Земли, $R$ - ее радиус. Чем выше мы поднимаем массу $m$, тем больше ее потенциальная энергия. При переходе к космическим масштабам

$$
g(r)=G \frac{M}{r^{2}}, R \leq r<+\infty .
$$

Рассмотрим две точечные массы $M$ и $m$. Согласно закону Ньютона две силы их взаимного притяжения действуют на эти две массы в противоположных направлениях вдоль прямой, проходящей через эти точки, при этом силы равны друг другу и выражаются формулой

$$
F(r)=G \frac{M m}{r^{2}}
$$

Сейчас мы хотим выяснить, как потенциальная энергия их взаимодействия зависит от $r$. Предположим, что расстояние между двумя массами увеличилось от $r$ до $r+\Delta r$. Расстояние увеличилось, конечно, не само по себе, а массу $m$ отдалили от массы $M$, которая словно гвоздем прибита к своему месту. Впрочем, можно говорить и наобо- рот, что массу $M$ отдалили от $m$, тогда гвоздем прибита масса $m$. В любом случае сторонняя сила совершила работу. Легко доказать, что эта работа не зависит от траектории отдаляемой массы. Для этого достаточно приближенно заменить кривую ломаной линией, состоящей из бесконечно малых отрезков, лежащих на прямых, проведенных через точки $m$ и $M$, и отрезков, перпендикулярных к ним. Работа совершается только на первых, а значит, ее можно найти по формуле

$$
\begin{aligned}
& A(r, r+\Delta r)=\int_{r}^{r+\Delta r} G \frac{M m}{x^{2}} d x= \\
& \quad=\left(-G \frac{M m}{x}+c\right)_{x=r}^{x=r+\Delta r}=G \frac{M m}{r(r+\Delta r)} \cdot \Delta r .
\end{aligned}
$$

Видно, что совершенная работа зависит только от начального и конечного значений расстояния между $m$ и $M$, а значит, сила всемирного тяготения потенциальна и потенциальная энергия задается формулой

$$
E_{\text {п }}(r)=-G \frac{M m}{r}+c,
$$

где $c$ - произвольная постоянная. Примечательно, что при стремлении $r$ к нулю работа по отдалению одного тела от другого на фиксированное расстояние $\Delta r$ растет неограниченно (для точечных масс).

Здесь уместно вспомнить фразеологизм для танго нужны двое. Объясним, почему. Пусть несколько точек лежат на одной прямой. Будем интересоваться расстояниями между точками из каждой их пары. Если точек две, то в школе советуют взять линейку, ее ноль совместить с одной из них и посмотреть, какое число соответствует второй точке. А можно вообще о нуле не думать, а просто-напросто совместить линейку с прямой, содержащей все эти точки. Тогда расстояние между двумя любыми точками есть модуль разности соответствующих им чисел. Это расстояние не зависит от расположения линейки, ее можно как угодно сдвигать влево-вправо вдоль прямой.

Говоря о расстоянии, всегда надо иметь две точки. Так же, говоря о потенциальной энергии гравитации тела, расположенного 
в данной точке, следует понимать, с какими другими телами оно гравитационно взаимодействует. В случае двух тел мы уже записывали формулу для $E_{\text {п }}(r)$. Эта формула задает бесконечное множество функций $E_{\Pi_{c}}(r)$. При этом для любых двух значений аргумента $a$ и $b$ разность соответствующих значений любых двух функций оказывается одной и той же:

$$
E_{\Pi_{c_{1}}}(a)-E_{\Pi_{C_{1}}}(b)=E_{\Pi_{c 2}}(a)-E_{\Pi_{c 2}}(b) .
$$

Следовательно, эта функция может служить для описания потенциальной энергии одной материальной точки относительно другой.

В физике для описания потенциальной энергии двух точечных масс выбрали функцию

$$
E_{\text {пा }}(r)=-G \frac{M m}{r} .
$$

Выбор основан на понятном условии, что когда тела не взаимодействуют (т.е. при $r \rightarrow+\infty)$, то их потенциальная энергия взаимодействия стремится к нулю. При этом любое значение потенциальной энергии оказывается отрицательным. Это может показаться противоречащим здравому смыслу. Но к этому надо привыкнуть, а главное, понять. Ведь действительно, если $r_{1}<r_{2}$, то $E_{\text {п }}\left(r_{1}\right)<E_{\text {п }}\left(r_{2}\right)$. Чем дальше (выше) от $M$, тем больше потенциальная энергия массы $m$.

Заметим, что наша формула также определяет и энергию взаимодействия $E_{12}$ двух шаров с массами $M$ и $m$, расстояние между центрами которых равно $r$, если плотность внутри каждого шара зависит только от расстояния до его центра. Чтобы избавиться от энергии взаимодействия $E_{12}$, надо удалить тела друг от друга на бесконечное расстояние, т.е. совершить работу

$$
A=G \frac{M m}{r} \text {. }
$$

При этом каждый шар имеет и собственную гравитационную энергию, связанную со взаимным притяжением его отдельных частей. Эти энергии определяются формулами

$$
E_{1}=-\omega_{1} G \frac{M^{2}}{R_{1}}, E_{2}=-\omega_{2} G \frac{m^{2}}{R_{2}},
$$

где числа $\omega_{1}$ и $\omega_{2}$ порядка 1 , они зависят только от распределения плотностей $\rho_{1}\left(r_{1}\right)$ и $\rho_{2}\left(r_{2}\right)$ внутри тел. Если мы хотим растащить каждое из тел на очень малые части и разместить их очень далеко друг от друга, то в этом случае нужно совершить работы $A_{1}=-E_{1}, A_{2}=-E_{2}$ против сил тяготения.

\section{Незнайка на Луне и модель Роша}

Самая мощная в мире электростанция находится в Китае и называется «Три ущелья». Она использует кинетическую энергию падающей воды, которая, в свою очередь, получается из гравитационной потенциальной энергии. Общеизвестная формула $E=m g h$ говорит, что энергия тем больше, чем больше будут три величины: масса воды $m$, высота ее падения $h$ и ускорение силы тяжести $g$.

В обычной жизни мы можем искать только наилучшие географические условия, т.е. наибольший поток воды и наибольший перепад высот. Но давайте предположим, что и третий фактор, т.е. ускорение $g$, также поддается оптимизации. Вспомним сказку Николая Носова «Незнайка на Луне» и смелое предположение Знайки о пустотелой Луне. Если видимая поверхность нашего спутника представляет собой тонкую, но жесткую оболочку радиусом $R_{1}$, а обитатели Луны живут на ее ядре радиусом $R_{2}$ с массой $M$, то каждая масса $m$, находящаяся на оболочке, может отдать при спуске на ядро весьма существенную энергию

$$
E=E_{\Pi_{1}}-E_{\Pi_{2}}=\left(-\frac{G M m}{R_{1}}\right)-\left(-\frac{G M m}{R_{2}}\right) .
$$

Пусть вся масса $M \approx 7,3 \cdot 10^{22}$ кг у такой фантастической Луны сосредоточена в ее ядре, $R_{1} \approx 1740$ км - радиус ее внешней оболочки, а, например, $R_{2}=\frac{1}{2} R_{1}-$ радиус обитаемого ядра. Тогда для груза массой 100 кг, находящегося на поверхности незнайкиной Луны, эта энергия равна $E \approx 2,8 \cdot 10^{8}$ Дж, что в 2800 раз больше, чем у 100 кг воды, падающей с высоты 


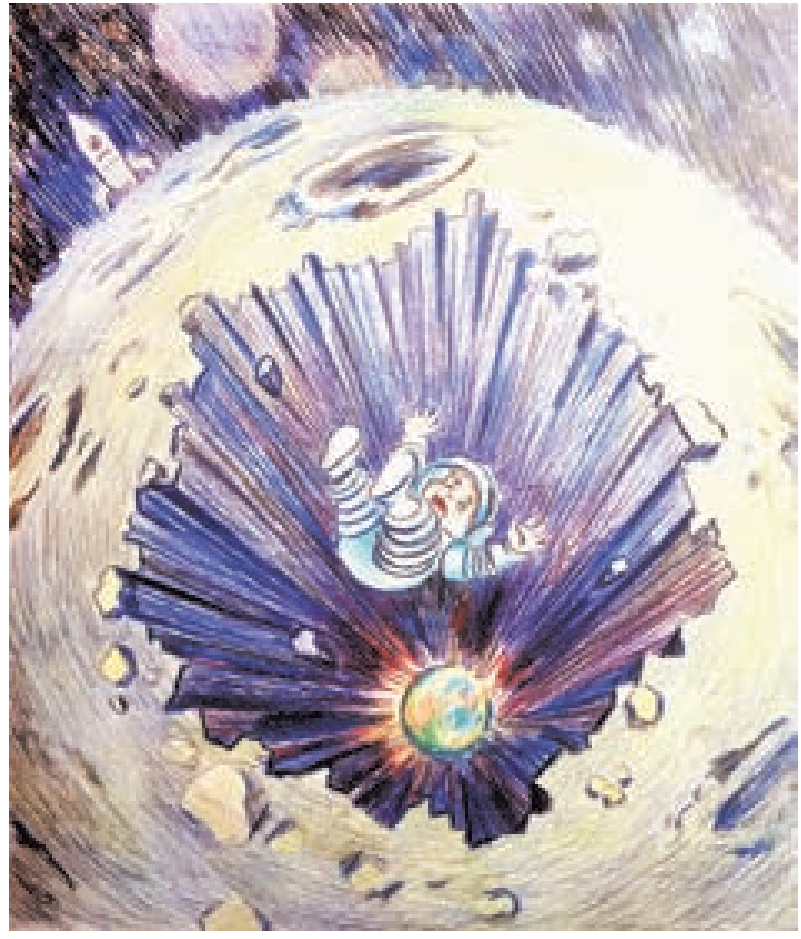

расстоянии от материальной точки, в которой сосредоточена масса звезды, при падении этого тела на центр будет бесконечно большим. Положение спасает общая теория относительности, в ней каждой массе соответствует ее гравитационный радиус и поэтому точечных масс не существует. Найти гравитационный радиус можно, как ни странно, из «скрещивания» закона всемирного тяготения и постулата специальной теории относительности о том, что двигаться в пустоте быстрее скорости света нельзя.

Для нахождения гравитационного радиуса вспомним вывод второй космической скорости $v_{2}$. Так называется минимальная скорость, которую надо придать телу, находящемуся на поверхности Земли, для его удаления от Земли на сколь угодно большое расстояние. При этом не учитывается ни наличие у Земли атмосферы, ни ее вращение вокруг своей оси, ни воздей-

100 м на Земле. Незнайка и Козлик могли бы продавать акции не только Компании Гигантских Растений, но и Компании Гигантских Энергий.

Нас могут упрекнуть в несерьезности, однако модель пустотелой звезды встречается не только в сказках. Есть она и в астрофизике и называется моделью Роша.

Эдуард Альбер Рош (1820-1883), французский астроном и математик, изучил предельный случай распределения массы внутри звезды, когда масса сосредоточена в ее центре. Пусть радиус звезды $R$, а радиус ее центра, т.е. ядра, $r$. Для Солнца у нас раньше получилось, что плотность в центре примерно в 100 раз больше средней. А модель Роша для Солнца дает, что радиус его ядра должен быть примерно в 5 раз меньше радиуса Солнца. Эта модель неплохо описывает распределение плотности в звездах. Рош также предложил закон изменения плотности Земли с глубиной. Из формулы потенциальной энергии, очевидно, следует, что для предельного случая модели Роша выделение энергии точечным телом, находящимся на конечном ствие других небесных тел, например Солнца. Кинетическая энергия тела на бесконечном расстоянии от Земли полностью переходит в потенциальную энергию:

$$
\frac{m v_{2}^{2}}{2}=\frac{G M m}{R},
$$

откуда

$$
v_{2}=\sqrt{\frac{2 G M}{R}},
$$

где $M$ - масса Земли, $R$ - ее радиус. Напомним, что при перемещении массы $m$ с орбиты радиусом $R$ в бесконечность ее потенциальная энергия получает приращение $\frac{G M m}{R}$. Полагая теперь для произвольной массы $M$ вторую космическую скорость (ее еще называют параболической скоростью) равной скорости света $c$ (ибо это согласно специальной теории относительности самая большая возможная скорость), мы найдем минимальный возможный радиус для этой массы:

$$
R_{g}=\frac{2 G M}{c^{2}} .
$$


Он и называется гравитационным радиусом. Ничто (даже свет!) не может покинуть поверхность тела массой $M$ с таким радиусом. Если тело не имеет материальной поверхности, то оно называется черной дырой и гравитационный радиус определяет так называемый горизонт черной дыры.

Модифицированная модель Роша - это черная дыра, которая окружена материальной сферой малой массы и весьма большого радиуса (очень большого по сравнению с гравитационным). Немного фантазии, и мы поселим на этой поверхности разумную цивилизацию. Тогда у этой «поверхностной» цивилизации не будет никаких проблем с энергией: достаточно проковырять дыру в поверхности и опускать туда на весьма длинной веревке, намотанной на ротор турбины, ненужные предметы (черная дыра будет еще и безотказным мусорным полигоном). А они при падении будут разгоняться до скорости света и вращать турбины электростанций. Тело массой $m$ в итоге даст нам энергию порядка $\frac{1}{2} m c^{2}$, больше получить никак невозможно, но и не нужно - вспомните, сколько мусора ежедневно принимают наши мусорные полигоны. Одна беда - воздух тоже будет втягиваться черной дырой...

При превращении звезды в черную дыру может выделиться энергия порядка $\frac{1}{2} M c^{2}$, т.е. примерно половина полной энергии звезды, включающей энергию покоя. Такое выделение сравнимо с энергией аннигиляции, при которой вещество полностью превращается в излучение, но для аннигиляции звезд нужно иметь антизвезду, т.е. звезду из антивещества, а их во Вселенной пока не обнаружили.

Если вернуться к самому началу времен, к Большому взрыву, то появляется мысль, что вечный двигатель все же существует и это сама Вселенная. Могла ли наша Вселенная родиться из ничего? Закон сохранения энергии не запрещает превращения нуля в сумму двух величин: положительной и отрицательной. Энергия гравитации отрицательна, энергия вещества - положительна. Значит, материя и пространство-время могут возникнуть одновременно. Но как же из этих крошечных новорожденных вырастает такой огромный мир? Здесь в роли волшебника выступает вакуум. Согласно квантовой теории, у вакуума есть положительная энергия и эта энергия создает отрицательное давление - антигравитацию, силу отталкивания, поэтому случайно родившийся мир начинает безумно быстро раздуваться. Отрицательность гравитационной энергии оказывается Вселенной только на руку. Но этот вакуум, обладающий большой плотностью энергии, неустойчив и превращается через какие-то $10^{-30}$ секунды в элементарные частицы, причем возможно, что всех таких частиц мы пока и не знаем, поскольку энергии наших ускорителей никак не дотягивают до тогдашних. Но плотность энергии вещества (частиц) быстро падает с расширением Вселенной, и даже раньше чем через секунду после Большого взрыва физика становится почти понятной! Почти - это значит, что мы знаем, как ведет себя 5\% «населения» Вселенной. Все же темная материя (25\%) и темная энергия (70\%) пока остаются для нас загадками... (O физике Вселенной в целом можно прочитать в статье авторов «Космология Фридмана: горы реальные и потенциальные» в «Кванте» №1, 2 за 2017 г.)

\section{Зажигаем звезды}

Пусть вокруг небесного тела массой $M$ вне его атмосферы обращается по круговой орбите радиусом $r$ со скоростью $v$ спутник массой $m$. Его центростремительное ускорение $a=\frac{v^{2}}{r}$ определяется силой взаимного притяжения $G \frac{M m}{r^{2}}$. Согласно второму закону Ньютона,

$$
m \frac{v^{2}}{r}=G \frac{M m}{r^{2}}
$$

тогда

$$
v^{2}=G \frac{M}{r} .
$$

Из последнего равенства следует, в частности, что если спутник в результате воз- 
действия на него следов атмосферы или после торможения двигателями оказался на более низкой орбите, то его скорость увеличилась.

Подсчитаем полную энергию спутника как сумму кинетической и потенциальной:

$$
\begin{aligned}
E=E_{\text {к }}+E_{\text {п }} & =\frac{m v^{2}}{2}-G \frac{M m}{r}= \\
& =m G \frac{M}{2 r}-G \frac{M m}{r}=-\frac{1}{2} G \frac{M m}{r} .
\end{aligned}
$$

Видим, что сохраняющаяся полная энергия спутника отрицательна и равна половине его гравитационной энергии. Это так называемая теорема вириала для спутника. Последнее уравнение можно записать так:

$$
E_{\mathrm{\kappa}}+E_{\text {п }}=\frac{1}{2} E_{\text {п }} \cdot
$$

Интересно, что похожая теорема вириала работает и внутри звезд. Кинетическую энергию спутника заменим кинетической энергией частиц идеального газа, который моделирует звездное вещество. На деле этим веществом является плазма, состоящая из заряженных частиц, но для звезд Главной последовательности, находящихся в равновесном состоянии, модель вполне пригодна. Теперь кинетическая энергия - это тепловая (внутренняя) энергия. А что происходит при сжатии звезды? Поскольку работу совершает сила гравитации, то гравитационная энергия уменьшается. Но она отрицательна. Это значит, что ее абсолютная величина растет. Тогда тепловая (т.е. кинетическая) энергия возрастает, и температура внутри звезды увеличивается. Тепловая энергия передается от внутренних слоев звезды наружу и излучается звездой в пространство. При этом звезда не остывает, а напротив, разогревается. Принято говорить, что звезда имеет отрицательную теплоемкость.

Если звезда не сжигает термоядерное горючее (такова судьба звезд с малой массой, температура в их центре мала для осуществления термоядерной реакции), то она может светить за счет постепенного сжатия, т.е. за счет гравитационной энер- гии. При этом светимость звезды $L$ определяется уменьшением ее полной энергии за единицу времени:

$$
L=-\frac{\Delta E}{\Delta t}=-\frac{1}{2} \frac{\Delta E_{\mathrm{II}}}{\Delta t} .
$$

При этом

$$
\frac{\Delta E_{\mathrm{K}}}{\Delta t}=-\frac{1}{2} \frac{\Delta E_{\mathrm{\Pi}}}{\Delta t}=L,
$$

т.е. внутренняя (или тепловая) энергия звезды возрастает на столько же, сколько излучает звезда наружу. Гравитационная энергия расходуется поровну между нагреванием внутренности звезды и излучением в окружающее пространство.

Как рождаются звезды? Отрицательность гравитационной энергии приводит к неустойчивости материи (а это, в основном, газообразный водород, 25\% гелия, 1\% дейтерия и ничтожно малая примесь легких элементов - лития, бериллия и бора), первоначально заполняющей Вселенную почти равномерно. Более плотные участки начинают забирать материю у соседей (первоначальное накопление капитала в экономике), неравенство непрерывно нарастает, но пока оно еще не так велико, существенна роль случайностей (мелкие предприниматели то богатеют, то разоряются). Однако в итоге появляются настолько большие центры уплотнения (сверхмонополии), что судьба их окружения оказывается решенной: начинается «падение на центр», гравитационный коллапс, приостановить который может только... сила Архимеда, т.е. возрастающее давление звездного вещества. Но с ростом давления растет и температура - и вот рождается звезда, горячее тело начинает светиться. Когда температура в центре звезды превысит 10 миллионов градусов, там начинаются термоядерные реакции, приводящие к превращению водорода в гелий. Именно этот источник энергии обеспечивает таким звездам, как Солнце, долгую счастливую жизнь (около 10 миллиардов лет). 